\title{
A NOVEL MOBILE ECG SENSOR WITH WIRELESS POWER TRANSMISSION FOR REMOTE HEALTH MONITORING
}

\author{
Jin-Chul Heo, Eun-Bin Park, Chan-Il Kim, \\ Hee-Joon Park and Jong-Ha Lee \\ Department of Biomedical Engineering, School of Medicine, \\ Keimyung University, Daegu, Korea
}

\begin{abstract}
For electromagnetic induction wireless power transmission using an elliptical receiving coil, we investigated changes in magnetic field distribution and power transmission efficiency due to changes in the position of the transmitting and receiving coils. The simulation results using the high-frequency structure simulator were compared with the actual measurement results. It has been shown that even if the alignment between the transmitting coil and the receiving coil is changed to some extent, the transmission efficiency on the simulator can be maintained relatively stable. The transmission efficiency showed the maximum when the center of the receiving coil was perfectly aligned with the center of the transmitting coil. Although the reduction in efficiency was small when the center of the receiving coil was within $\pm 10 \mathrm{~mm}$ from the center of the transmitting coil, it was found that the efficiency was greatly reduced when the receiving coil deviated by more than $10 \mathrm{~mm}$. Accordingly, it has been found that even if the perfect alignment is not maintained, the performance of the wireless power transmission system is not significantly reduced. When the center of the receiving coil is perfectly aligned with the center of the transmitting coil, the transmission efficiency is maximum, and even if the alignment is slightly changed, the performance of wireless power transmission maintains a certain level. This result proposes a standardized wireless transmission application method in the use of wireless power for implantable sensors.
\end{abstract}

\section{KEYWORDS}

ECG, Implantable sensors, Simulation, Power transmission efficiency, Wireless power transmission

\section{INTRODUCTION}

Wireless power transmission devices have been applied to many medical devices that can be inserted into the human body Inductively coupled wireless power transmission using electromagnetic induction is increasingly being applied to medical electronic devices[1-3]. There is an increasing demand for small electronic devices such as microneural stimulators, cochlear implants, and pacemakers[4-6]. As the size of the device decreases, there is a need to minimize the volume by reducing the size of the coil for power transmission. Therefore, realizing an efficient coil structure within a limited area is a very important task in wireless power transmission[7, 8].

Although various wireless power transmission technologies have been developed so far, they have not yet been commercialized except for some non-contact induction coupling methods. In Dhinaharan Nagamalai et al. (Eds): CSEIT, WiMoNe, NCS, CIoT, CMLA, DMSE, NLPD - 2020 pp. 17-27, 2020. CS \& IT - CSCP 2020 
the past, some studies have been made to use microwaves, such as $5.8 \mathrm{GHz}$, to transmit large powers of several tens of watts or more, but they are not actively commercialized due to the influence on the human body and the directivity due to the use of high efficiency antennas [9-11]. Particularly, the wireless transmission system that can be used for human body has little progress in development due to the problem of safety due to electromagnetic waves of wireless transmission. Wireless charging using magnetic induction phenomenon is performed in a short distance of a few millimeters, and it is possible to use a small-sized device of $3 \mathrm{~W}$ or less, and it is known that it can be applied to a human body by use of relatively low energy. However, the charging efficiency is extremely low due to a short receiving distance and a large amount of heat $[12,13]$.

The purpose of this study is to develop a wireless sensor that can be used in human body and applied it to wireless electrocardiogram. The wireless ECG sensor is a power supply method by the wireless power transmission technology through electromagnetic induction between a pair of coils. The transmitting coil and the receiving coil form a pair, and the electromotive force induced in the receiving coil by the magnetic field generated when the current is supplied to the transmitting coil supplies the DC power to the circuit connected to the receiving coil. In this process, we will evaluate the theoretical considerations of wireless power transmission system and the power transmission characteristics through simulation[14].

In this study, the theoretical considerations of the wireless power transmission system and the power transmission characteristics were evaluated through simulation.

\section{Material AND Method}

\subsection{System mode}

The magnetic field analysis is determined by the size and shape of the transmitting and receiving coils. Induction current and voltage are evaluated according to the gap between the transmitting coil and the receiving coil. Computer analysis simulations were performed using the finite element method (FEM).
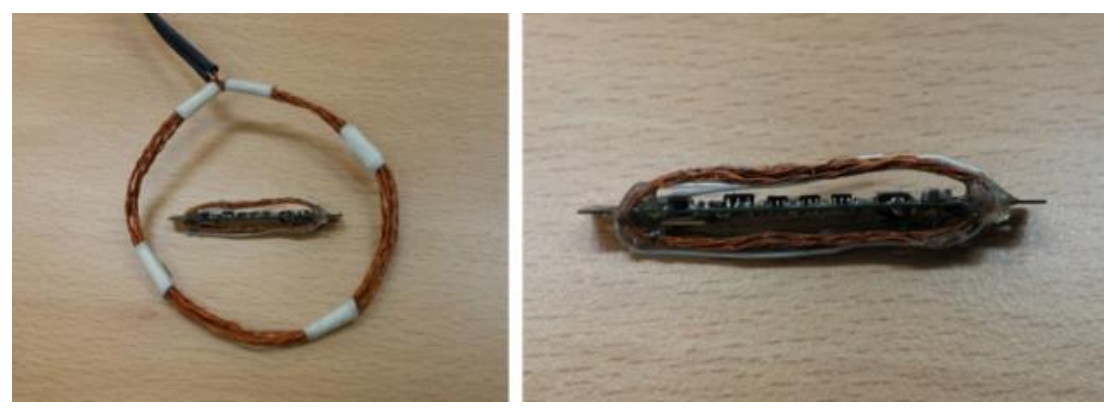

Figure 1. Round transmitting coil (Tx coil, left) and elliptical receiving coil (Rx coil, right). 


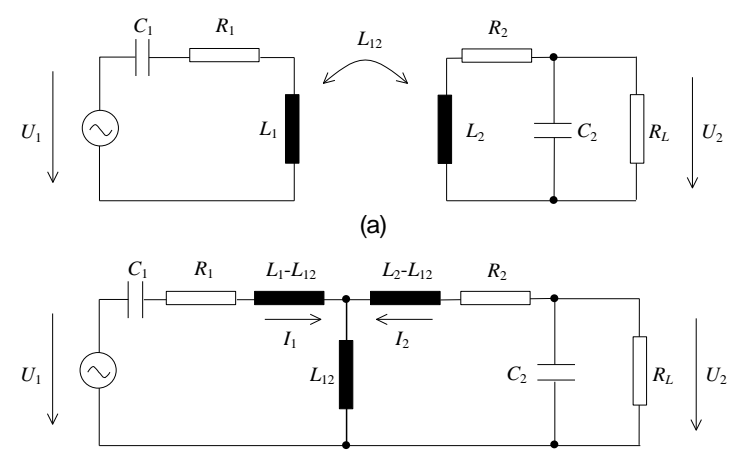

(b)

Figure 2. Wireless power transmission system. The circuit in which the transmitting coil (L1) and the receiving coil (L2) are combined is the equivalent circuit of the transmitting and receiving coils (L1-L2).

Transmitting coil (L1) and receiving coil (L2) use capacitance for L-C resonance to simulate electromagnetic induction in wireless power transmission. Capacitance $\mathrm{C} 1$ was connected in series to L1, and capacitance C2 was connected in parallel to L2. The total resistance of the transmit and receive coils and circuits is R1 and R2 (Figure 1 and 2). The two circuits that are magnetically coupled appear as a coupling factor that normalizes the mutual inductance or the mutual inductance for each coil. The delivered power can be calculated by the power delivery efficiency and the load of the receiving circuit.

$$
k=\frac{L_{12}}{\sqrt{L_{1} L_{2}}}
$$

In general, the power transfer efficiency that is widely used when expressing the power transfer performance is defined as follows.

$$
\eta=\frac{P_{L}}{P_{S}}
$$

At this time, the power PL transmitted to the load of the receiving circuit can be obtained as follows.

$$
P_{L}=\frac{V_{p k}^{2}}{2 R_{L}}
$$

\subsection{FEM modelling}

ANSYS Company's High Frequency Structure Simulator (HFSS) was used for finite element analysis of electromagnetic fields. HFSS can be used to simulate electromagnetic fields and electronic circuits in all frequency domains. Therefore, the electromagnetic field generated by the coil can be simulated even if the coil for wireless power transmission is connected to the L-C resonant circuit. The simulation shows the phenomenon that the transmitting and receiving coils resonate at $13.56 \mathrm{MHz}$, and the degree of activity of the magnetic field formed inside the transmitting and receiving coils. In addition, the evaluation of the voltage and power of the receiving coil is confirmed by the distance between the transmitting coil and the receiving coil, and the alignment between the transmitting coil and the receiving coil. 


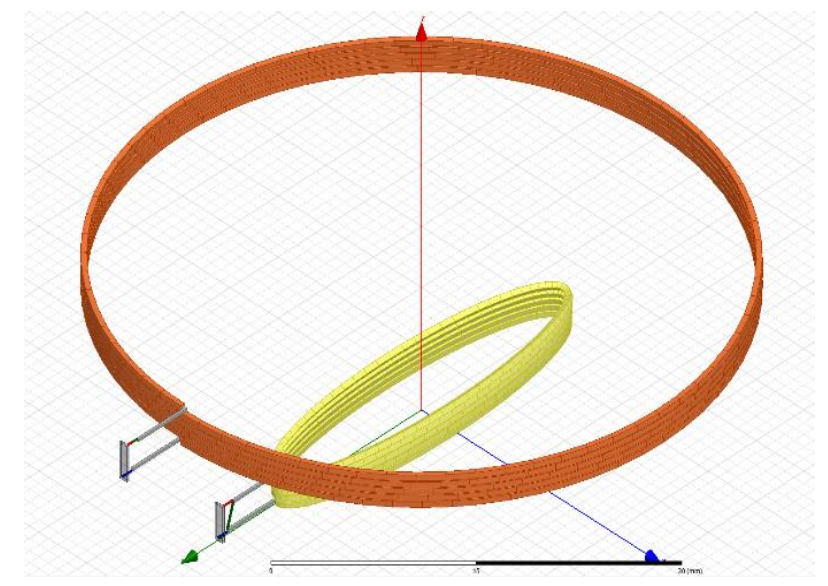

Figure 3. Modeling the transmitting coil (orange) and receiving coil (yellow).

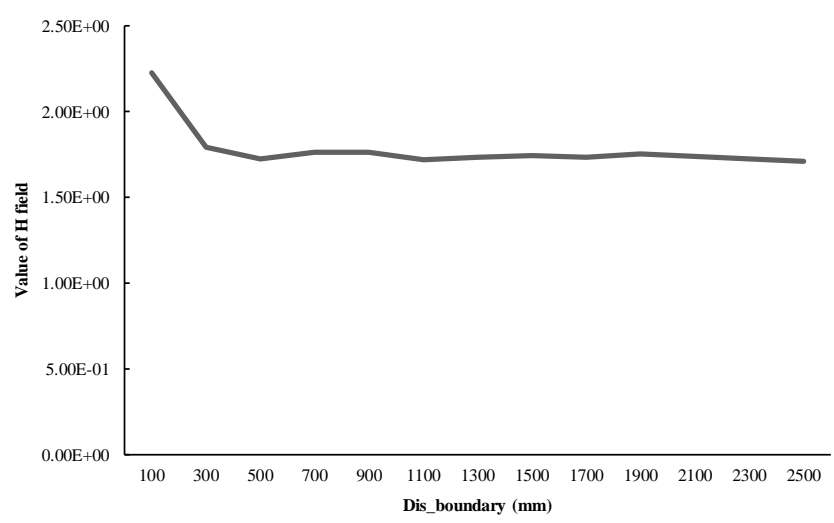

Figure 4. Convergence test for magnetic field strength as the size of the system simulation increases.

Wires with a circular cross section diameter of $0.5 \mathrm{~mm}$ require a long time to generate mesh and simulation of FEM. Therefore, you can convert a circular cross section into a rectangular cross section of the same area to model the wire as a rectangle with a length of $0.44 \mathrm{~mm}$.

The transmitting coil (Tx coil) is a $5 \mathrm{~cm}$ round coil, modeled in the form of a 5 th winding, and the receiving coil (Rx coil) is modeled as a 5th winding with an elliptical coil with a long axis of $3 \mathrm{~cm}$ and a short axis of $0.7 \mathrm{~cm}$. Orange indicates the transmitting coil and yellow indicates the receiving coil (Figure 3).

While determining the size of the space for simulation, by increasing the boundary between the magnetic field strength and the inductance value at a specific location, even if the boundary increases, the value of the magnetic field strength does not change. Inductance convergence is set as the optimal boundary that satisfies both efficiency and accuracy. The size suitable for simulation was determined to be $500 \mathrm{~mm}$ (Figure 4). 

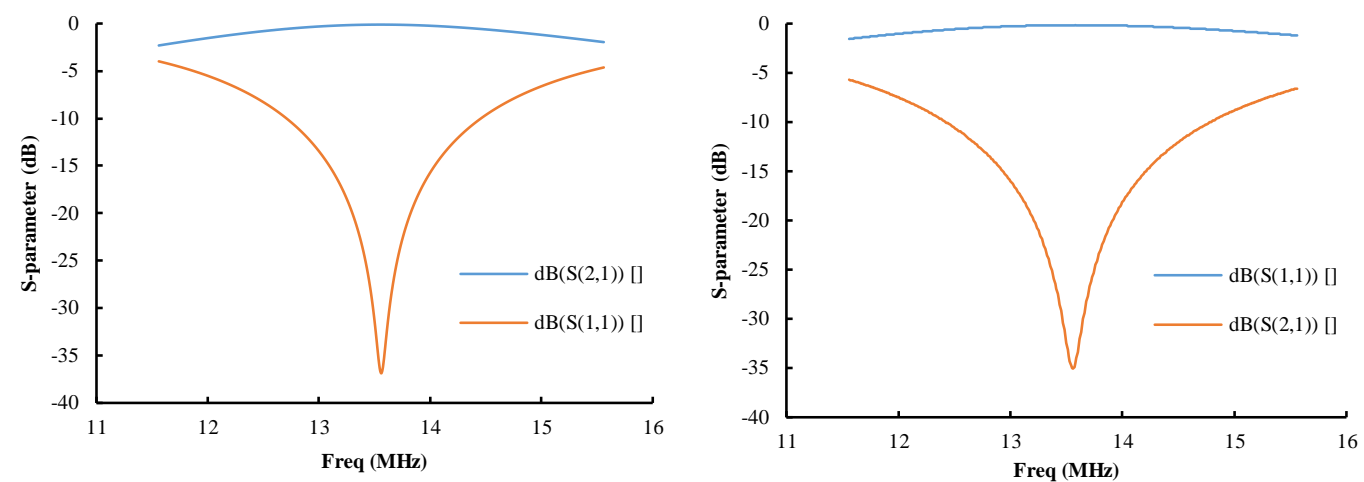

Figure 5. 13.56MHz resonance check for transmit (orange) coil (top) and receive (yellow) coil (bottom).

In order to attach a capacitor for resonating at $13.56 \mathrm{MHz}$ to each of the transmitting coil and the receiving coil, a two-port model was selected among the options available in HFSS. After calculating the resonant capacitance from $f=\frac{1}{2 \pi \sqrt{L C}}$ to ${ }^{C=\frac{1}{(2 \pi f)^{2} L}}$, based on the calculated value, the capacitance value was changed little by little to find the correct capacitance value at which resonance occurred at the desired resonant frequency (Figure 5).

\subsection{Simulation and verification of ECG sensor}

The distribution of magnetic field (H-field) is as follows when $13.56 \mathrm{MHz} \mathrm{AC}$ current is applied to the transmitting coil and the receiving coil is located at a certain distance on the $\mathrm{Z}$ axis. In addition, the power transmission efficiency was determined according to the relative position of the transmit / receive coils as well as the formed magnetic field(Figure 6).

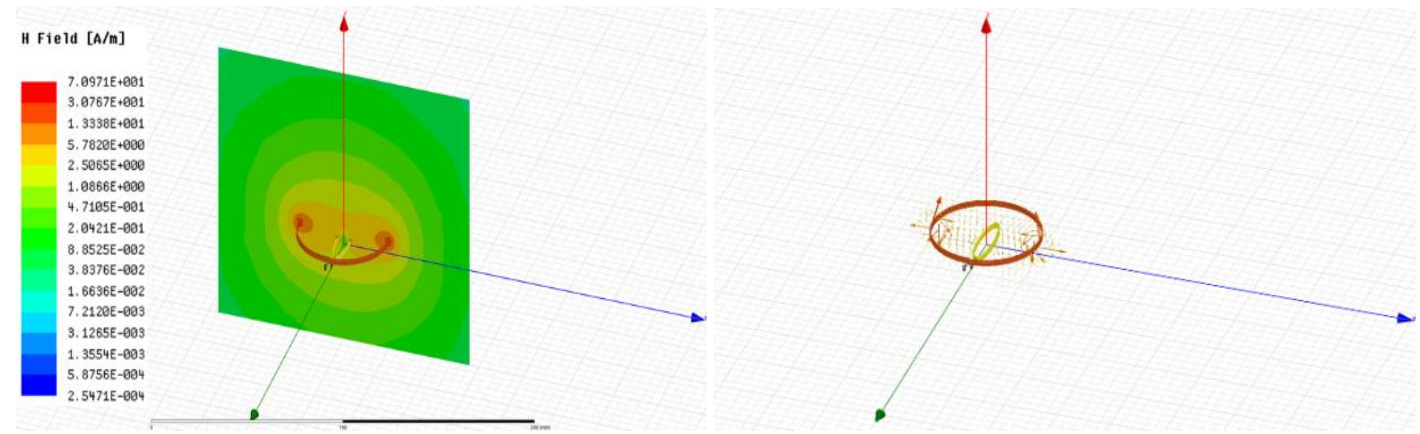

Figure 6. Distribution of magnetic field formed when $13.56 \mathrm{MHz}$ alternating current flows through the transmitting coil: (top) contour plot, (bottom) vector plot. 

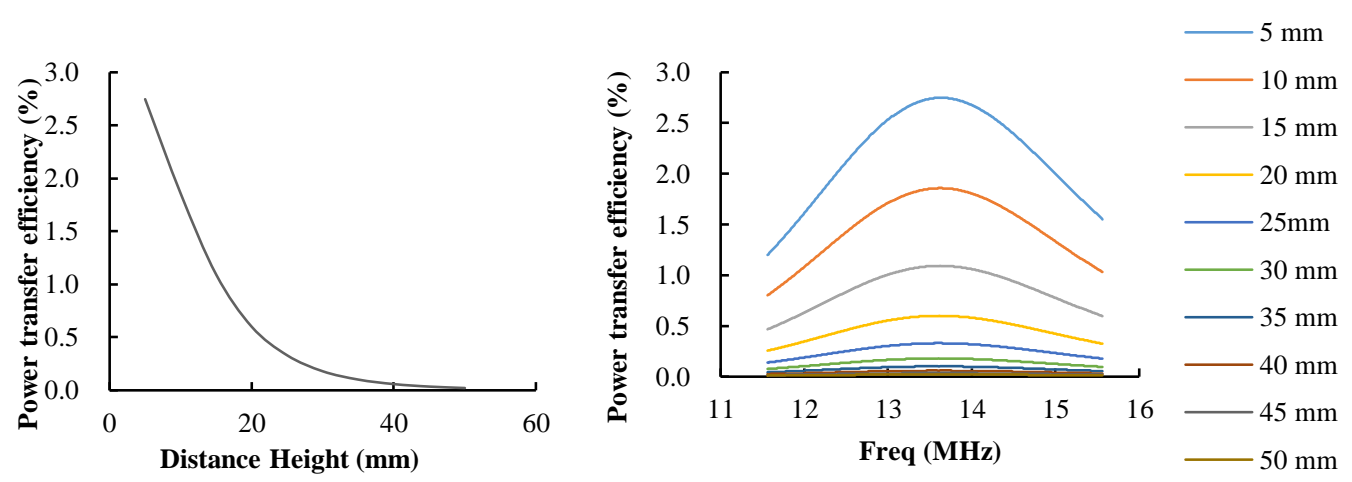

Figure 7. Changes in power transmission efficiency as the distance between the transmitting coil and the receiving coil increases (top) and frequency (bottom)

The simulation results show that the power transmission efficiency at each distance. When the distance between coils is $5 \mathrm{~mm}$ (minimum distance in the graph below), the transmission efficiency is about $2.74 \%$. The power transmission efficiency according to the frequency change around $13.56 \mathrm{MHz}$. It has the maximum transmission efficiency at the resonance frequency of the transmitting / receiving coil, and the transmission efficiency is decreased at a frequency other than the resonance frequency (Figure 7).

\subsection{Variations due to misalignment when coil-to-coil misalignment}

The change in the magnitude of the magnetic field in the case where the center of the receiving coil and the transmitting coil are perfectly aligned (misalignment is zero), the transmission coil is shifted $5 \mathrm{~mm}$ in the major axis direction of the ellipse until it becomes $25 \mathrm{~mm}$. The change of the power transmission efficiency when the receiving coil is moved in the major axis direction and the alignment turn aside. At this time, the distance between the transmitting and receiving coils in the Z-axis direction was fixed to $5 \mathrm{~mm}$. In a perfectly aligned state, the transmission efficiency is about $1.85 \%$, and the transmission efficiency is decreased as the degree of misalignment increases. However, there is no significant difference in transmission efficiency until the alignment is changed by about $10 \mathrm{~mm}$ (Figure 8 ).

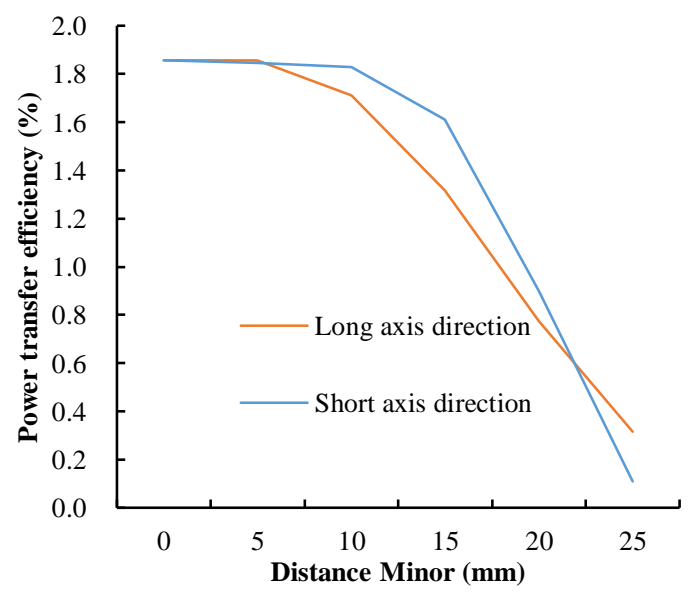

Figure 8 . When the alignment of the transmitting coil and the receiving coil is different in the long/short axis direction of the receiving coil. 


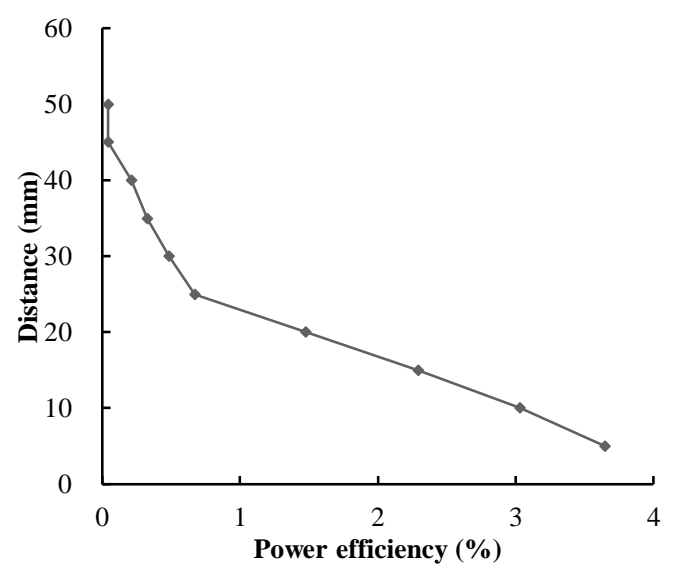

Figure 9. Power transmission efficiency.

The change of the magnetic field by the alignment was changed moving the transmission coil by $0 \mathrm{~mm}$ to $25 \mathrm{~mm}$ in the minor axis direction of the ellipse. The change in power transfer efficiency when the receiving coil was moved in the uniaxial direction and the alignment is wrong. The distance in the Z-axis direction between the transmitting and receiving coils is fixed at $5 \mathrm{~mm}$, and the transmission efficiency is the highest in a perfectly aligned state. As the degree of misalignment increases, the transmission efficiency decreases. However, it can be seen that there is no significant difference in transmission efficiency (Figure 9).

\subsection{Verification through experiments}

In order to verify the wireless power transmission through the simulation, we performed actual experiments. For the experiment, a transmission coil with a circular shape with a diameter of 5 $\mathrm{cm}$ and an elliptical shape with a long axis/short axis of $30 \mathrm{~mm} / 7 \mathrm{~mm}$ were fabricated. Both the transmitting and receiving coils were coated with copper wire having a diameter of $0.5 \mathrm{~mm}$, and the number of turns of the coils was set to 5 times. The inductance of the fabricated coil was measured to be $2.8 \mu \mathrm{H}$ for the transmit coil and $0.6 \mu \mathrm{H}$ for the receive coil. The resonant capacitance used to resonate the transmit and receive coils at $13.56 \mathrm{MHz}$ was $37 \mathrm{pF}$ and $228 \mathrm{pF}$, respectively.

This is an experiment in which the voltage value obtained at the receiving coil is measured while the distance between the coils is changed while the input to the transmitting coil is constant using the transmitting/receiving coil. And the result of measuring the voltage and power obtained from the receiving coil according to the distance between the coils. The power transmission efficiency was calculated as described.

Transmission efficiency: $\eta(\%)=(\mathrm{PL} / \mathrm{PS}) * 100$

The power at the load of the receiving coil: $\mathrm{PL}=(\mathrm{Vpk}) 2 / 2 \mathrm{RL}$

Power in the transmit coil: Ps $=$ Vs $*$ Is 
Table 1. Power transmission efficiency.

\begin{tabular}{ccccccc}
\hline $\begin{array}{c}\text { Distance } \\
(\mathrm{mm})\end{array}$ & $\begin{array}{c}\mathrm{Vp} \\
(\mathrm{V})\end{array}$ & VR_Tx $(\mathrm{V})$ & $\begin{array}{c}\text { Is } \\
(\mathrm{A})\end{array}$ & $\begin{array}{c}\text { Ps } \\
(\mathrm{W})\end{array}$ & PL(W) & $\begin{array}{c}\text { Power efficiency } \\
(\%)\end{array}$ \\
\hline 5 & 7.3 & 1.46 & 0.146 & 0.73 & 0.026645 & 3.65 \\
10 & 6.7 & 1.48 & 0.148 & 0.74 & 0.022445 & 3.033108108 \\
15 & 5.9 & 1.52 & 0.152 & 0.76 & 0.017405 & 2.290131579 \\
20 & 4.8 & 1.56 & 0.156 & 0.78 & 0.01152 & 1.476923077 \\
25 & 3.28 & 1.6 & 0.16 & 0.8 & 0.005379 & 0.6724 \\
30 & 2.8 & 1.62 & 0.162 & 0.81 & 0.00392 & 0.483950617 \\
35 & 2.3 & 1.62 & 0.162 & 0.81 & 0.002645 & 0.32654321 \\
40 & 1.84 & 1.6 & 0.16 & 0.8 & 0.001693 & 0.2116 \\
45 & 0.82 & 1.62 & 0.162 & 0.81 & 0.000336 & 0.041506173 \\
50 & 0.85 & 1.62 & 0.162 & 0.81 & 0.000361 & 0.044598765 \\
\hline
\end{tabular}

As a result, when the distance between the transmitting and receiving coils is $5 \mathrm{~mm}$, the voltage of the receiving coil (using $1 \mathrm{k} \Omega$ ) was $7.3 \mathrm{~V}$, the power was $26.6 \mathrm{~mW}$, and the power transmission efficiency was $3.6 \%$. When the distance between the coils is $20 \mathrm{~mm}$, the voltage, power, and transmission efficiency at the rod of the receiving coil were $4.8 \mathrm{~V}, 11.5 \mathrm{~mW}$, and $1.5 \%$, respectively. If the actual load (IC chip of various amplifiers) to which the receiving coil will transmit power requires $3.3 \mathrm{Vdc}$ operating power, if a full-wave rectifier is used to convert the induced AC voltage to DC voltage, When the input exceeds $4.8 \mathrm{~V}$, the $3.3 \mathrm{~V}$ DC voltage can be generated sufficiently. In other words, if you use the same coils as those used in this measurement, you can conclude that the transmit and receive coils can supply enough voltage and power even when they are $2 \mathrm{~cm}$ apart.

\subsection{In vivo model validation}

Fiteen healthy adult male SD rat (Sprague-Dawley rats) weighing 350-390 g were implanted with ECG sensors, and activated. The sensor between the peritoneal epithelium and the skin tissue, and approximately four weeks were allowed for the wounds at the surgical site to heal.

The wireless power system received signals from the sensor containing the ECG electric power supply, to transmit to an external monitor via Bluetooth. The display system consisted of an ECG signal output using a smartphone. The experiments were performed in accordance with the guidelines outlined in the Declaration of Helsinki, and were approved by the Ethic Committee of Keimyung University (Approval number, KM-2015-20R1). The transplanted ECG sensor showed a normal signal and confirmed that the experimental animal showed a pattern similar, less than $5 \%$ difference, to the simulation result of the wireless transmission in the fixed state (Figure 10).

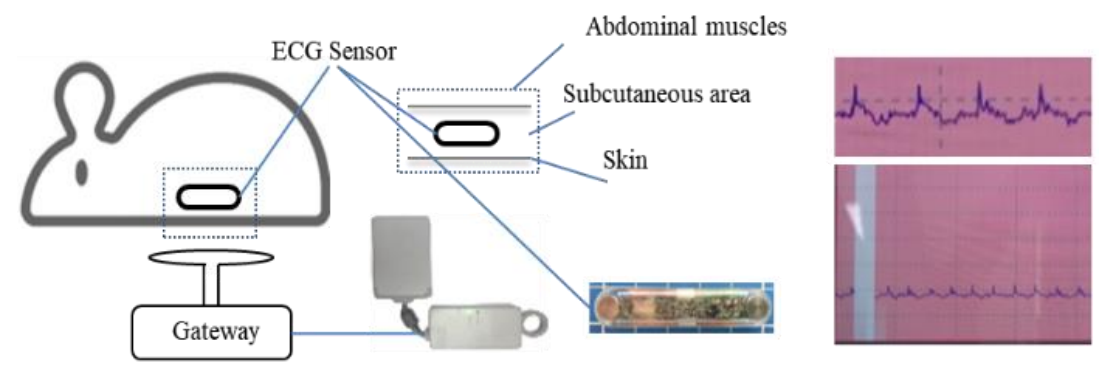

Figure 10. ECG sensor verification using in vivo model and wireless signal transmission. 


\section{DisCUSSION}

As the population ages and the welfare increases, research on implantable medical devices is being actively conducted, and products that provide more diverse functions are being released. On the other hand, the power consumption of implantable medical devices is increasing due to various functions, it is not possible to supply sufficient power with only the primary battery. In this study, the efficiency of a wireless power transmission system using a magnetic induction method was investigated. Computer modeling was used to verify the effectiveness of ECG detector power transmission, and to determine the applicability of the human body using a magnetic induction wireless power transmission system, we applied it to animal models using mice. As the required functions of implantable devices are diversified, power usage time is shortened and the restart cycle for power replacement is shortened, which increases the additional cost and physical and psychological burden. The development of a sustainable power module that reduces the cost and psychological burden of these additional surgeries is an essential technology enabling functional implementation of medical devices for human implantation[15-17].

The market for implantable medical devices is growing rapidly. Implantable medical devices are increasingly being applied to various disease treatment fields to help human weak functions, and power modules are increasingly needed for active treatment through devices. As research on wireless power transmission technology has been actively conducted, many attempts have been made to apply a method using electromagnetic waves, such as an electromagnetic induction technique or a magnetic resonance method, which is commercialized in electronic products to human insertion devices. However, the low-frequency method has a low absorption rate of the human body, a short transmission distance, and the high-frequency method has a high absorption rate of the human body, causing a temperature increase in skin tissue[18, 19].

The magnetic induction wireless transmission system used in this study receives electric induction between the transmitter coil and the receiver coil. When a magnetic field is generated in the transmitter coil, the receiver coil receives the magnetic field to induce electric power. This method has a high transmission efficiency of $90 \%$ or more, but the transmission distance is very short in several millimeters, and if the centers of the coils are not aligned with each other, the transmission efficiency is greatly reduced. However, it is known to be most suitable for application in the medical field due to its high safety and efficiency compared to the magnetic resonance method and electromagnetic wave method[20]. The results of this study can be used as a model of a power transmission system such as a human implant sensor using magnetic induction. The limitations of this study did not reflect animal movement for in vivo transplantation. In order to effectively apply the results of this study, it is necessary to verify the wireless power transmission efficiency for the movement of the detector.

IEC TC106 discusses the evaluation and measurement methods of electromagnetic human body exposure to radio power, and implantable medical devices have become a big issue in terms of safety[21-23]. Various wireless charging techniques may fail to reach commercialization because of possible hazards to the human body. High frequency and high power can induce human injury in implant application, too low output causes problems in operation of implant. In order to solve such a problem, it is possible to operate at low power and an electromagnetic wave environment within the human body protection standard is required. The development of miniaturization with lower power and the development of low-power-based implants is needed. In addition, strong electric fields and magnetic fields can lead to malfunctions of active implant devices such as artificial heart pacemakers, artificial eyes, and artificial heart, and problems must be solved[2426]. 
In this study, we investigated the distribution of the magnetic field and the power transmission efficiency according to the relative position between the transmitting and receiving coils when the electromagnetic induction type wireless power transmission is implemented using the elliptical receiving coil through the simulation study. If the coil is wound five times with the coil, the voltage that the coil can transfer to the load is more than $4.8 \mathrm{~V}$, which is enough to drive the IC with $3.3 \mathrm{~V}$ operating voltage. And the transmission efficiency at this time is about $1.5 \%$.

It is necessary to develop a variety of implants that can operate at low voltage, and to study the resistance to near field of active implant devices such as pacemakers and artificial eyes that should be mounted in the human body and various studies on low frequency band interference.

\section{Conclusion}

Through the simulation study, we have investigated how the distribution of magnetic field and the power transmission efficiency of the magnetic field formed according to the relative position between the transmit / receive coils change when the electromagnetic induction type wireless power transmission is implemented using a receive coil having an elliptical shape. The simulation using HFSS is relatively consistent with the actual measurement results, and can be usefully used for wireless power transmission simulation. When the center of the receiving coil is perfectly aligned with the center of the transmitting coil, the transmission efficiency is maximum, and even if the alignment is slightly changed, the performance of wireless power transmission maintains a certain level.

\section{ACKNOWLEDGMENT}

This research was supported by Basic Science Research Program through the National Research Foundation of Korea(NRF) funded by the Ministry of Education (NRF-2017R1D1A1B04031182 and 2017R1D1A1B03031747), and this research was supported by a grant of the Korea Health Technology R\&D Project through the Korea Health Industry Development Institute (KHIDI), funded by the Ministry of Health \& Welfare, Republic of Korea (grant number: HI18C0575010018)and this research was supported by a grant of Innopolis Foundation (Biosignal authentication and abnormal sign detection system through multi-object detection).

\section{REFERENCES}

[1] K. Kim, B. Kim, and C. H. Lee, "Printing Flexible and Hybrid Electronics for Human Skin and EyeInterfaced Health Monitoring Systems," Advanced Materials, vol. 32, no. 15, 2020.

[2] Y. Chen, Y. Cheng, Y. Jie et al., "Energy harvesting and wireless power transmission by a hybridized electromagnetic-triboelectric nanogenerator," Energy and Environmental Science, vol. 12, no. 9, pp. 2678-2684, 2019.

[3] Q. S. Abdullahi, R. Joshi, S. K. Podilchak et al., "Design of a wireless power transfer system for assisted living applications," Wireless Power Transfer, vol. 6, no. 1, pp. 41-56, 2019.

[4] Y. Ben Fadhel, S. Ktata, S. Rahmani et al., "Near-field Wireless Power Transfer is a promising approach to Power-up Active Implants." pp. 399-404, 2020.

[5] K. Agarwal, R. Jegadeesan, Y. X. Guo et al., "Wireless Power Transfer Strategies for Implantable Bioelectronics," IEEE Reviews in Biomedical Engineering, vol. 10, pp. 136-161, 2017.

[6] T. Campi, S. Cruciani, F. Maradei et al., "Pacemaker Lead Coupling with an Automotive Wireless Power Transfer System," IEEE Transactions on Electromagnetic Compatibility, vol. 61, no. 6, pp. 1935-1943, 2019.

[7] R. Chávez-Santiago, J. Wang, and I. Balasingham, "The ultra wideband capsule endoscope." pp. 7278, 2013. 
[8] G. Ahmed Zeeshan, R. Sundaraguru, and F. Naaz, "Wearable wireless sensor system with RF remote activation for industrial applications," International Journal of Recent Technology and Engineering, vol. 8, no. 3, pp. 4716-4720, 2019.

[9] M. Wang, J. Chen, X. Cui et al., "Design and Fabrication of 5.8GHz RF Energy Harvesting Rectifier." Wireless Power Transfer, vol. 6, on. 1, pp. 41-56, 2019.

[10] S. Suganuma, D. Hung Nguyen, Y. Nishioka et al., "The Logistics System by Rotary Wing Unmanned Aerial Vehicle with 28GHz Microwave Power Transmission." pp. 413-416, 2019.

[11] S. Almorabeti, M. Hanaoui, M. Rifi et al., "Microstrip patch antennas at 5.8GHz for wireless power transfer system to a MAV." ACM International Conference Proceeding Series, 2017.

[12] N. Khan, H. Matsumoto, and O. Trescases, "Wireless Electric Vehicle Charger with Electromagnetic Coil-Based Position Correction Using Impedance and Resonant Frequency Detection," IEEE Transactions on Power Electronics, vol. 35, no. 8, pp. 7873-7883, 2020.

[13] S. A. Sis, and H. Akca, "Maximizing the efficiency of wireless power transfer systems with an optimal duty cycle operation," AEU - International Journal of Electronics and Communications, vol. $116,2020$.

[14] K. Wang, L. Sang, Y. Zhang et al., "Optimization Design for Wireless Power Transfer System under the Variation of Load and Coupling Coefficients." pp. 867-872, 2019.

[15] T. Ghomian, and S. Mehraeen, "Survey of energy scavenging for wearable and implantable devices," Energy, vol. 178, pp. 33-49, 2019.

[16] A. Koruprolu, S. Nag, R. Erfani et al., "Capacitive Wireless Power and Data Transfer for Implantable Medical Devices." 2018 IEEE Biomedical Circuits and Systems Conference, BioCAS 2018 Proceedings, 2018.

[17] E. Katz, "Implantable biofuel cells operating in vivo: Providing sustainable power for bioelectronic devices: From biofuel cells to cyborgs." Proceedings - 2015 6th IEEE International Workshop on Advances in Sensors and Interfaces, IWASI, pp. 2-13, 2015

[18] A. W. S. Putra, M. Tanizawa, and T. Maruyama, "Optical wireless power transmission using si photovoltaic through air, water, and skin," IEEE Photonics Technology Letters, vol. 31, no. 2, pp. 157-160, 2019.

[19] K. Takahashi, T. Yamada, and Y. Takemura, "Circuit parameters of a receiver coil using a wiegand sensor for wireless power transmission," Sensors (Switzerland), vol. 19, no. 12, 2019.

[20] K. Zhang, C. Liu, Z. H. Jiang et al., "Near-Field Wireless Power Transfer to Deep-Tissue Implants for Biomedical Applications," IEEE Transactions on Antennas and Propagation, vol. 68, no. 2, pp. 1098-1106, 2020.

[21] C. Xiao, K. Wei, D. Cheng et al., "Wireless charging system considering eddy current in cardiac pacemaker shell: Theoretical modeling, experiments, and safety simulations," IEEE Transactions on Industrial Electronics, vol. 64, no. 5, pp. 3978-3988, 2017.

[22] B. Cheng, Y. Chatzinoff, D. Szczepanski et al., "Remote acoustic sensing as a safety mechanism during exposure of metal implants to alternating magnetic fields," PLoS ONE, vol. 13, no. 5, 2018.

[23] A. C. Özen, B. Silemek, T. Lottner et al., "MR safety watchdog for active catheters: Wireless impedance control with real-time feedback," Magnetic Resonance in Medicine, vol. 84, no. 2, pp. 1048-1060, 2020.

[24] M. V. Tholl, A. Haeberlin, B. Meier et al., "An intracardiac flow based electromagnetic energy harvesting mechanism for cardiac pacing," IEEE Transactions on Biomedical Engineering, vol. 66, no. 2, pp. 530-538, 2019.

[25] D. B. Ahire, and V. J. Gond, "Wireless power transfer system for biomedical application: A review." ICEI 2017, pp. 135-140, 2018

[26] C. Kasia, A. Appannagari, A. Joshi et al., "Safety of wireless capsule endoscopy in patients with implantable cardiac devices,” JGH Open, vol. 4, no. 2, pp. 241-244, 2020.

(C) 2020 By AIRCC Publishing Corporation. This article is published under the Creative Commons Attribution (CC BY) license. 